\title{
Exact evaluation of the cutting path length in a percolation model on a hierarchical network
}

\author{
R. F. S. Andrade ${ }^{1}$ and H. J. Herrmann ${ }^{2,3}$ \\ ${ }^{1}$ Instituto de Física, Universidade Federal da Bahia, 40210-210, Salvador, Brazil \\ ${ }^{2}$ Computational Physics, IfB, ETH-Hönggerberg, Schafmattstraße 6, 8093 Zürich, Switzerland \\ ${ }^{3}$ Departamento de Física, Universidade Federal do Ceará, Campus do Pici, 60455-760, Fortaleza, Brazil
}

(Received 5 March 2013; published 15 April 2013)

\begin{abstract}
This work presents an approach to evaluate the exact value of the fractal dimension of the cutting path $d_{f}^{C P}$ on hierarchical structures with finite order of ramification. Our approach is based on a renormalization group treatment of the universality class of watersheds. By making use of the self-similar property, we show that $d_{f}^{C P}$ depends only on the average cutting path $(\mathrm{CP})$ of the first generation of the structure. For the simplest Wheastone hierarchical lattice (WHL), we present a mathematical proof. For a larger WHL structure, the exact value of $d_{f}^{C P}$ is derived based on a computer algorithm that identifies the length of all possible CP's of the first generation.
\end{abstract}

DOI: 10.1103/PhysRevE.87.042113

PACS number(s): 05.50.+q, 89.75.Hc, 64.60.ah

\section{INTRODUCTION}

The evaluation of the watershed of a rough landscape is of fundamental interest, impacting many different geographic aspects, starting from the very definition of national and regional limits between countries, to evaluating the destination of water, pollutants, and agricultural fertilizers washed out by rain. The challenge of presenting a general mathematical solution for the watershed given a general landscape is still open. Recent extensive numerical work provided some quantitative information characterizing some watershed features, such as the fractal dimension $d_{f}^{W S}$.

It was observed that the value $d_{f}^{W S} \simeq 1.21 \ldots$, which is obtained for an uncorrelated random landscape [1,2], has also been reported in the evaluation of the fractal dimension of other models, such as the set of cutting bonds below and the set of bridge bonds above the threshold, respectively, of a percolation problem on a two-dimensional lattice [3]. The same fractal dimension has also been obtained for the optimum path crack [4], the surface of the clusters in explosive percolation [5], the random fuse model in strong disorder [6], the shortest path in loopless percolation [7], and the random polymer in high disorder [8]. As far as we know, the quoted (or related) problems have never been the subject of an exact analysis, although it has been shown that they fulfill the Schramm-Loewner evolution (SLE) properties [9]. Therefore, the identification of a universal physical mechanism justifying the emergence of this particular $d_{f}$ value for these problems is an open challenge.

In this work, we present an exact evaluation of the fractal dimension of the cutting path length on two distinct Wheatstone hierarchical lattices (WHL's) (see Fig. 1). Hierarchical lattices have played an important role in the study of critical phenomena, since the results they produce can be regarded as approximations to models on Euclidian lattices within the framework of the Migdal-Kadanoff real space renormalization group [10-15]. The solution we provide follows steps similar to those used in the analysis of physical models on hierarchical structures with finite ramification order. We make use of the exact scale invariance of the geometrical construction to analyze successive generations $g$ of the model, requiring that the same expressions be valid for $g$ and $g+1$ provided the pertinent quantities are rescaled in the way dictated by the geometrical construction.

The WHL hierarchical structure is constructed by successively replacing a simple line segment between two root points (the $g=0$ generation) with a more complex structure consisting of a set of $p$ parallel branches, each one containing $b$ inner connections and $b-1$ sites. The inner sites in each neighboring parallel branch are also connected by a bond. As a result of this procedure, the lattice at the $g+1$ generation can also be obtained by substituting each of the bonds of the $g=1$ generation by a $g$ generation lattice. This way of building up the successive generations of the lattice will be adopted in the derivation of our main result. Let us remark that the choice $b=p=L$ adopted herein turns out to be the most relevant one for the purpose of comparison with the square lattice. Indeed, the $g=1 L$-WHL coincides with a rectangle with $N=L \times(L+1)$ sites and $2 L^{2}-1$ bonds provided all sites in the first and the $(L+1)$ th row are kept with the same boundary condition imposed on the WHL root sites. This amounts to keeping, for an Ising model, all spins in those rows in the same state as the root sites or, in the cutting path problem, to preserving all $L-1$ horizontal bonds in these rows, as will become clear in the next section.

The resulting self-similar graph has a fractal dimension $[16,17]$

$$
d_{f}^{W}=\log [2 L(L-1)+1] / \log L .
$$

In the current work, we consider $L=2$ and $L=3$ (see Figs. 1 and 2). For these cases, Eq. (1) leads to $d_{f}^{W} \simeq 2.322 \ldots$ and $d_{f}^{W} \simeq 2.335$... correspondingly. $d_{f}^{W}$ converges to 2 in a logarithmic way when $L \rightarrow \infty$. The maximal number of bonds $B$ and sites $N$ and the shortest distance between root sites $D$ depend on $L$ and $g$. When $L=2(L=3)$, they are given by $B_{g}=5^{g}\left(13^{g}\right), N_{g}=\left(5^{g}+3\right) / 2\left[\left(13^{g}+3\right) / 2\right]$, and $D_{g}=2^{g}\left(3^{g}\right)$. Finally, it is worth mentioning that the $L$-WHL is a self-dual hierarchical structure, in the sense that the dual of the basic unit is topologically identical to the original structure. This property has proven to be of relevance in the analysis of spin models. For instance, it ensures that the WHL critical temperatures coincide with those of the corresponding 


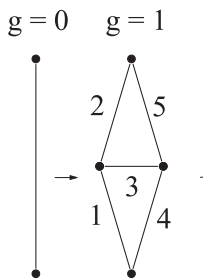

(a)

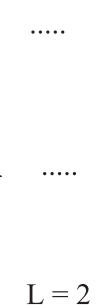

$\mathrm{L}=2$

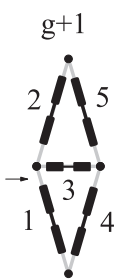

(b)
FIG. 1. (a) First steps of construction of the $L=2$ WHL, corresponding to $g=0$ and $g=1$. (b) Schematic construction of the $g+1$ lattice based on $5 g$ lattices, indicated by stylized dumbbells. Single bonds and dumbbells labeled from 1 through 5 are used in the proof of the main result in Sec. III.

models on square lattices, although the critical exponents are different [16].

\section{THE CUTTING BOND PATH}

The concept of cutting bond path $(\mathrm{CP})$ in a bond percolation model is defined for a dynamical process where existing bonds are randomly chosen and removed from the lattice. In this process, the order at which the bonds are removed is important, which turns the configuration space to grow as $B$ ! instead of the usual dependence $\sim \exp (B)$.

For the sake of definitiveness, let us consider the rectangle described above and start with a completely filled square lattice with $B=2 L^{2}-1$ bonds and $N=L \times(L+1)$. This configuration clearly allows for a connection between any site in the lowest row to any site in the upper most row.

Bonds are successively eliminated until the choice falls to a bond that if removed would disconnect the upper and lower rows. This bond is labeled as the cutting bond (in that particular removal sequence) and will be kept in the lattice, i.e., not removed, being the first element of the set of bonds in the CP. The removal process proceeds further, and a bond is only not removed whenever its elimination would lead to the disruption of the connection between the two boundary rows. When such an event occurs, the number of elements in the CP set is increased by one. The process is repeated until no other bonds but those labeled as cutting bonds remain in the lattice. The process that identifies the elements in the $\mathrm{CP}$

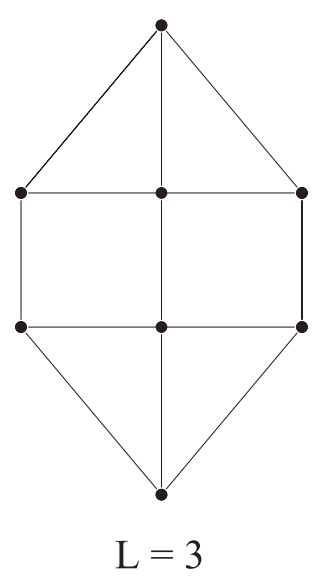

FIG. 2. First generation of the $L=3$ WHL. set ensures that they form a continuous line consisting of $\ell(i)$ bonds linking one site in the lowest row to one site in the upper most row. The index $i$ identifies the random sequence at which the bonds should be successively eliminated. We denote by $S$ the set of all distinct CP's. Note that a CP is dependent on the order at which the cutting bonds are assigned to it, so that $S$ contains a large number of elements that are formed by the same set of bonds. The average cutting path length is obviously

$$
\langle\ell\rangle=\frac{1}{\Omega(B)} \sum_{i=1}^{\Omega(B)} \ell(i)=\frac{1}{\Omega(B)} \sum_{i \in S} \ell(i),
$$

where $\Omega(B)=B$ ! counts the number of different sequences according to which the $B$ bonds are considered for elimination from the lattice. The fact that the value of $\Omega(B)$ increases faster than exponential is the essential difficulty to be overcome in an exact calculation of $\langle\ell\rangle$.

\section{THE WHL CUTTING PATH}

Let us consider the task of evaluating $\langle\ell\rangle$ for the WHL. For such structure, we have to start by considering all paths that connect one root site to the other. The random choice of the bonds to be eliminated follows the same prescription as for the square lattice, a selected bond becoming a cutting bond if its removal from the structure causes the connection between the two root sites to break.

The difficulty of this otherwise very hard task can be managed if we take advantage of the hierarchical structure of WHL and of the fact that its sites have a finite ramification order. The fact that, even in the limit $g \rightarrow \infty$, the WHL structure can be decoupled into more than one infinite component by removing a finite number of sites makes it possible to recursively evaluate $\left\langle\ell_{g}\right\rangle$ for any generation $g$. Let us consider the simpler case $L=2$ and proceed by mathematical induction over $g$ to prove that $\left\langle\ell_{g}\right\rangle=\left(\frac{34}{15}\right)^{g}$.

$$
\text { A. } L=2 \text { and } g=1
$$

Let us indicate by $S_{g}$ the set of all distinct CP's in the WHL at a given value of $g$. When $g=1$, the number of bonds is $B_{1}=$ 5 , so that there are $\Omega(5)=5 !=120$ sequences according to which the individual bonds can be eliminated. We start the computation of the $\mathrm{CP}$ lengths by noting that the 120 sequences can be cast into two distinct sets $\left(t_{1}\right.$ and $\left.a_{1}\right)$, according to whether the transverse bond is the first one to be eliminated or one of the four adjacent bonds to one root site is chosen first.

The set $t_{1}$ is such that, for all of its 24 different sequences, we obtain $\ell(i)=\ell_{g=1, t}=\left\langle\ell_{1, t}\right\rangle=2$. The set $a_{1}$ comprises 96 sequences but, due to the up-down and right-left symmetries of the WHL structure, it is necessary to compute only the subset of sequences starting with the elimination of the bond 1, according to the labeling indicated in Fig. 1. We let the partial average for the first elimination of an adjacent bond $\left\langle\ell_{1}(a)\right\rangle$ be written as

$$
\left\langle\ell_{1, a}\right\rangle=\frac{1}{4}\left[\ell_{1}(1,2)+\ell_{1}(1,3)+\ell_{1}(1,4)+\ell_{1}(1,5)\right],
$$

where the subscript 1 denotes the value of $g$ and $\ell_{1}(1, j)$ indicates the average CP length when the $j$ th bond is second one to be eliminated. When the bond 2 is eliminated in second place, the $\mathrm{CP}$ is formed by the bonds 4 and 5 , irrespective of 
the order of the elimination of the three remaining bonds. The same happens when the bond 3 is eliminated in second place, so that $\ell_{1}(1,2)=\ell_{1}(1,3)=2$ for all 6 sequences that enter in the formation of each partial average.

If the bond 4 is chosen to be the second one to be removed, the connection between the root sites would be broken at that step, so that the bond 4 is the first cutting bond for such sequences. Next, it is necessary to analyze in detail the third elimination. If either of the bonds 2 or 3 is chosen, then the CP length is 2 irrespective of the order of the remaining eliminations. If the bond 5 is eliminated in third place, the $\mathrm{CP}$ length is 3 , so that $\ell_{1}(1,4)=(3+2 \times 2) / 3$. Finally, if the bond 5 is eliminated in second place, the CP length is 3 for all 6 different sequences for eliminating bonds 2,3 , and 4 .

This leads to

$$
\left\langle\ell_{1, a}\right\rangle=7 / 3
$$

and, as a consequence,

$$
\left\langle\ell_{1}\right\rangle=\frac{1}{5}\left(\left\langle\ell_{1, t}\right\rangle+4\left\langle\ell_{1, a}\right\rangle\right)=\frac{34}{15} .
$$

Since the $g=0 \mathrm{WHL}$ consists of the single direct connection between the root sites, it is obvious that $\left\langle\ell_{0}\right\rangle=1$ and, as consequence, our statement is verified for $g=1$.

Before we proceed further with our proof, let us remind the reader that the $g=2$ WHL generation contains $B_{2}=25$ bonds, so that the number of different sequences over which the average of $\ell(i)$ has to be performed is $\Omega(25)=25 !>10^{25}$. This provides a flavor for the difficulties we would face in evaluating $\left\langle\ell_{g}\right\rangle$ if we could not use the arguments based on the geometric symmetry and finite ramification order.

\section{B. $L=2$ and $g>1$}

The second part of the proof depends essentially on the fact that the five $g$ lattices forming the $g+1$ lattice are separated by cutting sites, so that each $g$ unit is independent of the others. Figure 1(b) illustrates, in a schematic way, how the $g+1$ lattice is assembled by connecting five $g$ lattices, which are indicated by dumbbells. Next we note that once a cutting bond emerges in one of the $g$ lattices, the two root sites of that $g$ lattice will be connected by a part of the cutting path in the $g+1$ lattice. The opposite is also valid, in the sense that either the two root sites of a $g$ lattice are connected by the cutting path or there will be no cutting bond in that $g$ lattice. As long as the first cutting bond does not appear in the sequence of bond eliminations, the order at which the bonds are removed is only important within each $g$ lattice, and does not depend on the relative order of elimination among the five lattices. The order at which bonds are eliminated in other units only becomes relevant when two (or three) units contain at least one cutting bond. However, even in this situation, the form of the cutting path within one $g$ lattice does not depend on the order of the bond elimination in other $g$ lattices with cutting bonds.

Once these geometrical features have been clarified, we proceed with some mathematical rigor to complete the proof by assuming that $\left\langle\ell_{g}\right\rangle=\left(\frac{34}{15}\right)^{g}$. Our purpose is to show that $\left\langle\ell_{g+1}\right\rangle=\left(\frac{34}{15}\right)^{g+1}$, which requires that we adapt the counting process used in the previous subsection.
We start by noting that $\Omega\left(B_{g+1}\right)$, the number of different sequences in generation $g+1$, increases by a factor

$$
\frac{\left(5^{g+1}\right) !}{\left(5^{g}\right) !}=\prod_{k=5^{g}+1}^{k=5^{g+1}} k
$$

with respect to $\Omega\left(B_{g}\right)$. This very large factor is due to the fact that the random bond selection process does not require that each of the five generation $g$ lattices that are put together to build one generation $g+1$ lattice be sequentially emptied. If this were the case, this factor would simply be 5!. However, the identification of individual removal sequences is not necessary. In fact, it is sufficient to characterize the five sets $E_{g+1}(k), k=1,2,3,4,5$, respectively containing the removal sequences in which the root sites of the $k$ th $g$ lattice become disconnected in first place. Given the fact that all $g$ lattices are equivalent, it follows that $\mathcal{C}\left(E_{g+1}(k)\right)$, the number of elements (or cardinality) of each set $E_{g+1}(k)$, is the same for all values of $k$, which necessarily leads to $\mathcal{C}\left(E_{g+1}(k)\right)=\Omega\left(B_{g+1}\right) / 5$.

Now, it follows that there are again 5 ! different sequences along which the root sites of the five $g$ lattices become disconnected. They are exactly those identified in the previous subsection for removing the five bonds for the $g=1$ generation. Therefore, it is possible to cast all sequences into a finer classification than that given above, by assigning the order at which the root sites of each $g$ lattice become disconnected. We can use the same symmetry arguments used before to identify that there are, in fact, just two independent five step sequences: the $24 t$ sequences, at which the transverse $g$ lattice is the first one to be disconnected, and the $96 a$ sequences, in which a $g$ lattice sharing one of its root sites with the $g+1$ lattice is the first one to be disconnected. Note that the total number of removal sequences contained in the $24 t$ sequences is exactly $\Omega\left(B_{g+1}\right) / 5$. If we let $\left\langle\ell_{g+1, t}\right\rangle$ and $\left\langle\ell_{g+1, a}\right\rangle$ be the average lengths associated with the $t$ and $a$ sequences in the $g+1$ generation, it is possible to write

$$
\left\langle\ell_{g+1}\right\rangle=\frac{1}{5}\left(\left\langle\ell_{g+1, t}\right\rangle+4\left\langle\ell_{g+1, a}\right\rangle\right) .
$$

Let us evaluate $\left\langle\ell_{g+1, t}\right\rangle$. Since the transverse $g$ lattice has been disconnected in first place, it turns out that the $g+1 \mathrm{CP}$ is necessarily formed by bonds placed either in the 1 and $2 g$ lattices or in the 4 and $5 \mathrm{~g}$ lattices. Let us identify the sets of such sequences by $S_{g+1}(1,2) \subset S_{g+1}$ and $S_{g+1}(4,5) \subset S_{g+1}$. Since these are equivalent, we may concentrate, without loss of generality, on the CP's formed by sequences in $S_{g+1}(1,2)$. It is then clear that $\mathcal{C}\left(S_{g+1}(1,2)\right)=\Omega\left(B_{g+1}\right) / 10$, leading to

$$
\begin{aligned}
\left\langle\ell_{g+1, t}\right\rangle & =\frac{5}{\Omega\left(B_{g+1}\right)} \sum_{i \in E_{g+1}(3)} \ell_{g+1}(i) \\
& =\frac{10}{\Omega\left(B_{g+1}\right)} \sum_{i \in S_{g+1}(1,2)} \ell_{g+1}(i) .
\end{aligned}
$$

Next we make use of the fact that each $\mathrm{CP}$ in the set $S_{g+1}(1,2)$ can be separated in two parts, each of them formed, respectively, by bonds in the 1 and $2 g$ lattices. Since all CP's must go through a root site of both 1 and $2 g$ lattices, a CP in the set $S_{g}(1,2)$ can be obtained by the concatenation of two CP's of the generation $g$. Thus, $\ell_{g+1}(i)=\ell_{g, g+1}^{1}(i)+\ell_{g, g+1}^{2}(i)$, where $\ell_{g, g+1}^{j}(i)$, with $j=1$ and 2 , is the number of cutting bonds 
of the $g+1$ sequence $i$ in the $j$ th $g$ lattice. Therefore we can write

$$
\begin{aligned}
\left\langle\ell_{g+1, t}\right\rangle= & \frac{10}{\Omega\left(B_{g+1}\right)} \sum_{i \in S_{g+1}(1,2)} \ell_{g, g+1}^{1}(i) \\
& +\frac{10}{\Omega\left(B_{g+1}\right)} \sum_{i \in S_{g+1}(1,2)} \ell_{g, g+1}^{2}(i) .
\end{aligned}
$$

To evaluate the first sum in Eq. (8), we take into account the fact that it is possible to identify and separate the independent contributions depending on the removal sequence inside the $g$ lattices 1 only. Given the fact that the two sums are equivalent, the same procedure applies also for the second sum. Thus, select one removal sequence $i^{*} \in S_{g+1}(1,2)$ and follow the steps at which the specific bonds appear in the $1 g$ lattice. Repeat this procedure for each value of $i \leqslant \Omega\left(B_{g+1}\right) / 10$. During this process, it is possible to find a large number $\rho_{g+1}\left(1,2 ; i^{*}\right)$ of different CP's in the set $S_{g+1}(1,2)$, for which the subset of bonds in the $j=1 g$ lattice is the same as that in the $i^{*}$ path. Since this is true for any choice of the path $i^{*}$, we can simplify the notation and just write $\rho_{g+1}\left(1,2 ; i^{*}\right)=\rho_{g+1}(1,2)$.

Finally, as the 1 and $2 \mathrm{~g}$ lattices are equivalent, we must have $\rho_{g+1}(1,2)=\rho_{g+1}(2,1)=\Omega\left(B_{g+1}\right) / 10 \Omega\left(B_{g}\right)$. Therefore, Eq. (8) can be written as

$$
\begin{aligned}
\left\langle\ell_{g+1, t}\right\rangle= & \frac{1}{\Omega\left(B_{g}\right) \rho_{g+1}(1,2)} \sum_{i \in S_{g+1}(1,2)} \ell_{g, g+1}^{1}(i) \\
& +\frac{1}{\Omega\left(B_{g}\right) \rho_{g+1}(2,1)} \sum_{i \in S_{g+1}(1,2)} \ell_{g, g+1}^{2}(i) .
\end{aligned}
$$

Since the term in the first sum depends only on the bonds in the $1 g$ lattice, $\sum_{i \in S_{g+1}(1,2)} \ell_{g, g+1}^{1}(i)=$ $\rho_{g+1}(1,2) \sum_{i^{\prime} \in S_{g}} \ell_{g, g+1}^{1}\left(i^{\prime}\right)$, where we have used a new variable $i^{\prime}$ in the sum on the right-hand side to stress the fact that now the sum is performed over the CP's of the generation $g$. This property can be immediately used to simplify the second sum, in such way that

$$
\begin{aligned}
\left\langle\ell_{g+1, t}\right\rangle & =\frac{1}{\Omega\left(B_{g}\right)} \sum_{i^{\prime} \in S_{g}} \ell_{g, g+1}^{1}\left(i^{\prime}\right)+\frac{1}{\Omega\left(B_{g}\right)} \sum_{i^{\prime} \in S_{g}} \ell_{g, g+1}^{2}\left(i^{\prime}\right) \\
& =2 \frac{1}{\Omega\left(B_{g}\right)} \sum_{i^{\prime} \in S_{g}} \ell_{g, g+1}^{2}\left(i^{\prime}\right)=2\left\langle\ell_{g}\right\rangle
\end{aligned}
$$

Similar arguments can be used to evaluate $\left\langle\ell_{g+1, a}\right\rangle$. As we have shown in the former subsection, the CP's resulting from those removal sequences where the first pair of root sites of a $g$ lattice to be disconnected have a common root site with the $g+1$ lattice may have bonds in two or three different $g$ lattices. The proportion of paths in each situation is exactly the same as that obtained in Eq. (3), so that we obtain

$$
\left\langle\ell_{g+1, a}\right\rangle=7 / 3\left\langle\ell_{g}\right\rangle
$$

and, as a consequence,

$$
\left\langle\ell_{g+1}\right\rangle=\frac{34}{15}\left\langle\ell_{g}\right\rangle=\left(\frac{34}{15}\right)^{g+1} .
$$

The above arguments can be used whenever we consider exact self-similar structures with finite order of ramification. In such cases, the presence of cutting points in the $g+1$ lattice that are equivalent to the root sites of the $g$ lattice constitutes the key property that allows for similar proofs. Therefore, for this class of structures, the value of $\left\langle\ell_{g}\right\rangle$ depends only on the value of $\left\langle\ell_{g=1}\right\rangle$.

\section{Fractal dimension}

Since the typical length scale in the WHL is the shortest distance between the two root sites, it follows that the $\mathrm{CP}$ fractal dimension is given by

$$
d_{f}^{C P}=\frac{\log \left(\left\langle\ell_{g}\right\rangle\right)}{\log \left(D_{g}\right)}=\frac{\log (34 / 15)^{g}}{\log (2)^{g}} \simeq 1.1805
$$

for the $L=2$ WHL. This value is not far from the one found for $d_{f}^{C P}$ in the square lattice.

The evaluation of $d_{f}^{C P}$ for the $L=3 \mathrm{WHL}$ is much more difficult, although we actually need only to evaluate $\left\langle\ell_{g=1}\right\rangle$. The $g=1$ lattice has 13 bonds, hence $13 ! \sim 6 \times 10^{9}$ cutting paths should be identified. The evaluation of the $\left\langle\ell_{g=1}\right\rangle$ was parallelized and distributed into $25 \mathrm{Xeon}$ CPU cores, requiring $\sim 5$ days of CPU time for this task. The result we obtained,

$$
\begin{aligned}
d_{f}^{C P} & =\frac{\log \left(\left\langle\ell_{g}\right\rangle\right)}{\log \left(D_{g}\right)}=\frac{\log (35318809 / 9266400)^{g}}{\log (3)^{g}} \\
& \simeq 1.21791,
\end{aligned}
$$

is very close to the accepted value for $d_{f}^{C P}$ in the square lattice, which is $1.2168 \pm 0.0005$ [18].

Despite the hierarchical structure of the WHL family and the fact that the fractal dimension is slightly larger than two, it is interesting to note that the $d_{f}^{C P}$ values for two WHL's are comparable to those found on the square lattice. It should also be remarked that the next case $L=4$ has 25 connections when $g=1$. Note that $25 !>10^{25}$, and therefore it seems that the exact evaluation of $d_{f}^{C P}$ for larger values of $L$ is not feasible with current day computing facilities, at least within the framework developed herein.

\section{CONCLUSIONS}

We have presented the first renormalization group treatment for the fractal dimension of the cutting bond length, which is in the same universality class as the watershed, the optimum path crack, and the shortest path in loopless percolation. This calculation is in fact identical to the exact solution on a specific type of hierarchical lattice. Our result is particularly interesting because, as opposed to the usual problems in statistical physics, the phase space here grows like $N$ ! instead of exponentially because of the history dependence of the configurations. It would be interesting to apply in the future our technique to other problems involving ranked surfaces such as the sharing of reservoirs [19].

\section{ACKNOWLEDGMENTS}

The authors acknowledge support from the European Research Council (ERC), Advanced Grant No. 
319968-FlowCCS, from the Brazilian agencies FAPESB (Project No. PRONEX 0006/2009) and CNPq, and from the
Brazilian National Institute of Science and Technology of Complex Systems (INCT-SC).
[1] E. Fehr, J. S. Andrade, Jr., S. D. da Cunha, L. R. da Silva, H. J. Herrmann, D. Kadau, C. F. Moukarzel, and E. A. Oliveira, J. Stat. Mech. (2009) P09007.

[2] E. Fehr, D. Kadau, N. A. M. Araújo, J. S. Andrade, Jr., and H. J. Herrmann, Phys. Rev. E 84, 036116 (2011).

[3] K. J. Schrenk, N. A. M. Araújo, J. S. Andrade, Jr., and H. J. Herrmann, Sci. Rep. 2, 348 (2012).

[4] J. S. Andrade, Jr., E. A. Oliveira, A. A. Moreira, and H. J. Herrmann, Phys. Rev. Lett. 103, 225503 (2009).

[5] N. A. M. Araújo and H. J. Herrmann, Phys. Rev. Lett. 105, 035701 (2010)

[6] A. A. Moreira, C. L. N. Oliveira, A. Hansen, N. A. M. Araújo, H. J. Herrmann, and J. S. Andrade, Jr., Phys. Rev. Lett 109 255701 (2012).

[7] M. Porto, S. Havlin, S. Schwarzer, and Armin Bunde, Phys. Rev. Lett. 79, 4060 (1997).

[8] M. Cieplak, A. Maritan, and J. R. Banavar, Phys. Rev. Lett. 72, 2320 (1994).
[9] E. Daryaei, N. A. M. Araújo, K. J. Schrenk, S. Rouhani, and H. J. Herrmann, Phys. Rev. Lett. 109, 218701 (2012).

[10] A. A. Migdal, Zh. Eksp. Teor. Fiz. 69, 810 (1975) [Sov. Phys. JETP 42, 413 (1976)].

[11] A. A. Migdal, Zh. Eksp. Teor. Fiz. 69, 1457 (1975) [Sov. Phys. JETP 42, 743 (1976)].

[12] L. P. Kaddanoff, Ann. Phys. 100, 359 (1976).

[13] A. N. Berker and S. Ostlund, J. Phys. C 12, 4961 (1979).

[14] P. M. Bleher and E. Zälys, Commun. Math. Phys. 67, 17 (1979).

[15] M. Kaufman and R. B. Griffiths, Phys. Rev. B 24, R496 (1981).

[16] C. Tsallis and A. Magalhães, Phys. Rep. 268, 305 (1996).

[17] A. C. Almeida and R. F. S. Andrade, Physica A 357, 181 (2005).

[18] E. Fehr, K. J. Schrenk, N. A. M. Araújo, D. Kadau, P. Grassberger, J. S. Andrade, Jr., and H. J. Herrmann, Phys. Rev. E 86, 011117 (2012).

[19] K. J. Schrenk, N. A. M. Araújo, and H. J. Herrmann, Sci. Rep. 2, 751 (2012). 